\title{
Assessing the knowledge, attitude and practice of osteoporosis among Singaporean women aged 65 years and above at two SingHealth polyclinics
}

Dypti Lulla' ${ }^{1}$, MD, Chiang Wen $\underline{T e O}^{2}$, MB BCh BAO, MRCP, XiaoYou Shen ${ }^{1}$, MBBS, Zhi Bing Julian Loi ${ }^{1}$, MBBS, Khai Wen Quek ${ }^{2}$, MD, GDFM, Hosanna Liha Anak $\underline{\mathrm{Lis}}^{2}$, MB BCh BAO, Sheila Anthony $\underline{K o h}^{1}$, MBBs, Eric Tao $\underline{\text { Chan }}{ }^{2}$, MD, Sarah Woon Ching $\underline{\text { Lim }}^{1}$, MBBS, MMed, Lian Leng Low ${ }^{3}$, MBBS, MMed

\begin{abstract}
INTRODUCTION Singapore has one of the world's most rapidly ageing populations. Osteoporosis is associated with significant morbidity and mortality from hip fractures in the elderly. This pilot study aims to evaluate the knowledge, attitude and practice of osteoporosis among Singaporean women aged $\geq 65$ years, and assess barriers to osteoporosis screening. METHODS We conducted a cross-sectional survey of 99 English-speaking women aged $\geq 65$ years at two SingHealth polyclinics by convenience sampling. The validated Osteoporosis Prevention and Awareness Tool was used to assess their knowledge about osteoporosis prevention and awareness and perceived barriers to osteoporosis screening. Osteoporosis health education was provided, and bone mineral density (BMD) screening was offered to all participants.

RESULTS The response rate was $91.6 \%$. The majority of the participants (54.5\%) had low knowledge of osteoporosis, and only $12.1 \%$ had high knowledge scores. Higher education levels were associated with higher knowledge scores $(p=0.018)$. Although participants with higher knowledge scores were more willing to undergo osteoporosis screening, these findings did not reach statistical significance $(p=0.067)$. The top reasons for declining BMD testing were misconceptions that lifestyle management is sufficient to prevent osteoporosis, poor awareness and knowledge of the disease, and the perceived high cost of BMD testing.

CONCLUSION Interventions should focus on osteoporosis education and, eventually, BMD screening for less-educated patients. Health education should rectify common misconceptions of the disease, increase awareness of osteoporosis and improve screening rates.
\end{abstract}

Keywords: barriers, bone mineral density, knowledge, osteoporosis

\section{INTRODUCTION}

The proportion of Singapore residents aged $\geq 65$ years has increased from $8.8 \%$ in 2009 to $14.4 \%$ in 2019. ${ }^{(1)}$ The incidence of hip fractures in Singapore has also increased because of its ageing population. ${ }^{(2)}$ Hip fractures in the elderly are often fragility fractures secondary to undiagnosed osteoporosis. Bone remodelling is affected by the activity of bone formation by osteoblasts and bone resorption by osteoclasts. Ageing tilts this balance towards osteoclast activity and leads to a decrease in bone mass and weakening microarchitecture, resulting in osteoporosis and a higher risk of fragility fractures. Osteoporosis - in combination with other factors associated with ageing such as nutrition, physical activity, comorbid medical conditions and drugs - increases the risk of falls and fragility fractures. ${ }^{(3)} \mathrm{A}$ fragility fracture is defined as a fracture that occurs with minimal trauma, such as a fall from one's standing height or lower with no other identifiable major forces. ${ }^{(4)}$ Among osteoporosisrelated fragility fractures, hip fractures and vertebral fractures have the highest socioeconomic burden on society and lead to significant morbidity and mortality. Hip fractures are associated with increased risk of mortality within the first few years after the injury ${ }^{(5)}$ and can be a significant economic burden from acute inpatient management of hip fractures, rehabilitation after hospitalisation and additional fractures if osteoporosis is untreated. ${ }^{(6)}$ Higher mortality from hip fractures is noted in patients from lower socioeconomic status, elderly individuals with other medical conditions, delayed operations, those not taking antiosteoporosis drugs after the fracture and those with more severe fractures. With an increasingly ageing population and increasing incidence of hip fractures, timely screening for osteoporosis and appropriate, early treatment will reduce the risk of a fracture and fracture-related mortality and economic burden.

Although osteoporosis leads to deterioration in quality of life and quality-adjusted life-year, which improve with treatment, osteoporosis is unfortunately underdiagnosed and undertreated in Asia. Some of the reasons cited for underdiagnosis include inadequate dual-energy X-ray absorptiometry (DEXA) machines, lack of awareness among the public and professionals, the asymptomatic nature of osteoporosis, inaccessibility to care and high cost of diagnostics. Approximately $30 \%$ of the doctors were not aware of the guidelines for bone mineral density (BMD) testing, and less than $30 \%$ of doctors would perform BMD when faced with patients with fractures. ${ }^{(7)}$ A population-based survey of women aged $\geq 45$ years in Singapore demonstrated that only $58 \%$ of the sample had heard of osteoporosis; these women tended to be younger, better educated, have regular exercise, or are single. ${ }^{(8)}$

Various countries and societies such as the National Osteoporosis Foundation, ${ }^{(9)}$ United States Preventive Services

${ }^{1}$ SingHealth Polyclinics - Marine Parade, ${ }^{2}$ SingHealth Polyclinics - Sengkang, ${ }^{3}$ Department of Family Medicine and Continuing Care, Singapore General Hospital, Singapore Correspondence: Dr Dypti Lulla, Resident Physician, Family Medicine Service, KK Women's and Children's Hospital, 100 Bukit Timah Road, Singapore 229899. Dypti.lulla@singhealth.com.sg 
Task Force, ${ }^{(10)}$ and International Society for Clinical Densitometry (Asia Pacific consensus) ${ }^{(11)}$ have recommended a population-wide strategy for women aged $\geq 65$ years to undergo BMD screening using DEXA. Higher-risk populations below the recommended age can be identified using pre-screening tools such as the Osteoporosis Self-assessment Tool for Asians (OSTA) ${ }^{(12)}$ or the Fracture Risk Assessment Tool (FRAX) developed by the World Health Organization Collaborating Centre for Metabolic Bone Diseases at the University of Sheffield..$^{(13)}$

Osteoporosis awareness, diagnosis and management are important for early diagnosis and prevention of hip fractures and the associated morbidity, mortality and economic burden of treatment. In this pilot study, we evaluated the knowledge, attitudes and practices towards osteoporosis of Singaporean women aged $\geq 65$ years and how these are associated with willingness to screen for osteoporosis. We also assessed perceived barriers to undergoing osteoporosis screening in this population. Many factors contribute to the poor osteoporosis screening rate in our population. We hypothesised that lack of awareness and understanding of osteoporosis is a significant factor leading to low screening rates. In our study, we aimed to explore some of these factors so that targeted interventions can be designed to improve osteoporosis screening rates.

\section{METHODS}

We conducted a cross-sectional survey of 99 English-speaking women aged $\geq 65$ years at two SingHealth Polyclinics in Singapore, Polyclinic A and Polyclinic B. Polyclinics in Singapore are government subsidised primary care clinics. Women at the two polyclinics were selected by convenience sampling from the clinic waiting areas. Participants were recruited if they met the following inclusion criteria: female gender, aged $\geq 65$ years, ability to read English, having mental capacity and having the ability to provide informed consent. Those who had any cognitive impairment with a score $\leq 7$ on the Abbreviated Mental Test or those who were unable to read English were excluded from the study. The participants were offered a quiet space to complete the questionnaire. The study investigators were available to provide simple clarification if the participants had questions while doing the questionnaire, without prompting them with the answers.

The validated Osteoporosis Prevention and Awareness Tool (OPAAT) ${ }^{(14)}$ was used with permission from the authors to assess participants' knowledge and awareness about osteoporosis. A validated questionnaire was only available in English and had 30 questions assessing knowledge about osteoporosis pathophysiology, prevention and consequences of untreated disease. Surveys were graded as 1 point for each correct answer and 0 point for incorrect answers or if the participant chose the 'don't know' option. Score cut-offs were based on the original paper. A score of $\geq 24$ out of 30 on the OPAAT was considered good knowledge and a score of 19-23 was considered average knowledge. A score of $<19$ was considered low knowledge.

Post-secondary education was considered as higher education in our results. Patient barriers to osteoporosis screening were evaluated. Osteoporosis health education was provided, and
Table I. Key demographics of participants $(\mathbf{n}=99)$.

\begin{tabular}{|ll|}
\hline Characteristic & No. (\%) \\
\hline Age* $(\mathbf{y r})$ & $70(67-74)$ \\
\hline Ethnicity & \\
\hline Chinese & $81(81.8)$ \\
\hline Malay & $11(11.1)$ \\
\hline Indian & $6(6.1)$ \\
\hline Others & $1(1.0)$ \\
\hline Marital status & \\
\hline Single & $9(9.1)$ \\
\hline Married & $68(68.7)$ \\
\hline Separated & $1(1.0)$ \\
\hline Divorced & $8(8.1)$ \\
\hline Widowed & $13(13.1)$ \\
\hline Highest education level & \\
\hline No formal education & $2(2.0)$ \\
\hline Primary & $16(16.2)$ \\
\hline Secondary & $54(54.5)$ \\
\hline A-level/diploma (ITE/polytechnic/private school) & $16(16.2)$ \\
\hline University/post-tertiary & $11(11.1)$ \\
\hline Employment status & \\
\hline Employed & $25(25.3)$ \\
\hline Unemployed/looking for a job & $4(4.0)$ \\
\hline Retired/housewife & $70(70.7)$ \\
\hline OPAAT score & $33(33.3)$ \\
\hline Low knowledge (< 19) & $12(12.1)$ \\
\hline Average knowledge (19-23) & \\
\hline High knowledge (> 23) & \\
\hline & \\
\hline
\end{tabular}

*Data presented as median (interquartile range). ITE: Institute of Technical Education; OPAAT: Osteoporosis Prevention and Awareness Tool

BMD screening was offered to all participants. Information from the questionnaires was de-identified and transcribed into REDCap for ease of data analysis and assessed for completion. Data was analysed using chi-square analysis in IBM SPSS Statistics version 25.0 (IBM Corp, Armonk, NY, USA). A p-value $<0.05$ was considered statistically significant.

\section{RESULTS}

A total of 157 women were approached, of whom 119 met the eligibility criteria. Those who did not meet the criteria did not fulfil the age requirement and had limited English language skills. 108 women agreed to participate in the study, and 99 women completed the survey (response rate 91.6\%).

The majority of the participants were Chinese and married, with a median age of 70 years. The majority of the participants had completed secondary education and were retired or housewives (Table I). Our sample population was more educated compared to the general Singaporean population, as population demographics for Singaporean aged $\geq 55$ years demonstrated that almost $50 \%$ lacked secondary education. ${ }^{(15)}$

The majority of the participants (54.5\%) had a low knowledge score of $\leq 18$, and only $12.1 \%$ had high knowledge scores of $\geq 24$. The mean score was $17.6 \pm 4.9$, and the median score 
Table II. Breakdown of OPAAT scores by question, with correct answers in parenthesis.

\begin{tabular}{|c|c|c|}
\hline \multirow[t]{2}{*}{ Question } & \multicolumn{2}{|c|}{$\%$} \\
\hline & Correct & Incorrect \\
\hline 1. Makes bone weaker, more brittle and more likely to break (fracture). (True) & 88.9 & 11.1 \\
\hline 2. Everybody will get osteoporosis as it is part of ageing. (False) & 20.1 & 79.8 \\
\hline 3. Osteoporosis occurs because bone is removed faster than it is formed. (True) & 53.3 & 46.5 \\
\hline 4. Osteoporosis and osteoarthritis are different names we use to describe the same disease. (False) & 31.3 & 68.7 \\
\hline 5. Osteoporosis usually has no symptoms. (True) & 50.5 & 49.5 \\
\hline 6. Postmenopausal women are not at risk for osteoporosis. (False) & 69.7 & 30.3 \\
\hline 7. Osteoporosis is an untreatable disease. (False) & 55.6 & 44.4 \\
\hline 8. A bone mineral density test is used to diagnose osteoporosis. (True) & 64.6 & 35.4 \\
\hline 9. I do not need a bone mineral density test unless I fracture my bones. (False) & 67.7 & 32.3 \\
\hline 10. A bone mineral density test is high in radiation. (False) & 33.3 & 66.7 \\
\hline 11. A bone mineral density test should be performed monthly to monitor bone loss. (False) & 60.6 & 39.4 \\
\hline 12. Results in back pain. (True) & 55.6 & 44.4 \\
\hline 13. Loss of height or hunchback. (True) & 83.8 & 16.2 \\
\hline 14. Loss of mobility (unable to move around by myself). (True) & 75.8 & 24.2 \\
\hline 15. Results in tooth loss. (False) & 15.2 & 84.8 \\
\hline 16. Results in joint pain or swelling of fingers. (False) & 20.2 & 79.8 \\
\hline 17. The recommended daily intake for calcium in women above 50 years of age is $1,000 \mathrm{mg}$. (True) & 56.6 & 43.4 \\
\hline 18. It is too late to increase calcium intake after age 50. (False) & 50.5 & 49.5 \\
\hline 19. Glucosamine can help prevent osteoporosis. (False) & 32.3 & 67.7 \\
\hline 20. Calcium supplements can help prevent osteoporosis. (True) & 74.7 & 25.3 \\
\hline 21. The regular dose of calcium supplements can cause kidney stones. (False) & 29.3 & 70.7 \\
\hline 22. Food such as milk, tofu, anchovies (ikan bilis), yellow dhal and spinach are rich in calcium. (True) & 90.9 & 9.1 \\
\hline $\begin{array}{l}\text { 23. You can obtain your recommended daily intake of vitamin D via exposing your skin to sunlight } \\
\text { for about } 15 \text { minutes a day. (True) }\end{array}$ & 74.7 & 25.3 \\
\hline 24. Increasing coffee and tea intake can help in osteoporosis prevention. (False) & 64.6 & 35.4 \\
\hline 25. Weight-bearing exercise (such as brisk walking and line dancing) can decrease bone loss. (True) & 60.6 & 39.4 \\
\hline 26. Exercise will wear out bones. (False) & 78.8 & 21.2 \\
\hline $\begin{array}{l}\text { 27. Certain medications (such as sleeping tablets or high blood pressure medications) may reduce } \\
\text { the risk of falling. (False) }\end{array}$ & 51.5 & 48.5 \\
\hline 28. To prevent falls, comfortable shoes with a good grip should be used. (True) & 97.0 & 3.0 \\
\hline 29. Poor vision may lead to falls. (True) & 92.9 & 7.1 \\
\hline 30. Being underweight helps prevent osteoporosis. (False) & 59.6 & 40.4 \\
\hline
\end{tabular}

OPAAT: Osteoporosis Prevention and Awareness Tool

was 18 (interquartile range 14.5-21.0). The distribution of the scores and breakdown by responses are seen in Tables I and II, respectively. Higher education levels were associated with higher knowledge scores ( $p=0.018$; Table III). Although participants with higher knowledge scores were more willing to undergo osteoporosis screening, these findings did not reach statistical significance ( $p=0.067$; Table IV).

$42.4 \%$ of the participants had already undergone BMD screening. Among those who had never had BMD screening, $47.4 \%$ were willing to undergo screening, and $43.9 \%$ were agreeable to schedule it on the same day. The top reasons for declining BMD were misconceptions that lifestyle management (regular exercise and calcium intake) is sufficient to prevent osteoporosis, poor awareness and knowledge of the disease, and the perceived high cost of BMD screening. Other concerns cited include polypharmacy and risk of medication side effects. Similar concerns have been cited for poor adherence to osteoporosis treatment, ${ }^{(16)}$ including time-consuming follow-up appointments, medication costs and side effects, and the perceived lack of importance of osteoporosis treatment.

Incidentally, participants at Polyclinic A were more likely to have previously done BMD screening, compared to those at Polyclinic B $(60 \%$ vs. $21 \%$, p < 0.001). Education levels and knowledge scores were further evaluated by site to determine any difference in the populations that may support the higher screening rates noted at Polyclinic A. Although participants from Polyclinic A had higher education levels $(p<0.001)$, there was no significant difference in their knowledge of osteoporosis $(p=0.174)$.

\section{DISCUSSION}

We found that higher education levels corresponded to better knowledge of osteoporosis. The local population appeared to have better knowledge of lifestyle measures to prevent osteoporosis, falls prevention and some complications of osteoporosis. 
Table III. Correlation between education levels and knowledge of osteoporosis using the OPAAT.

\begin{tabular}{|llll|}
\hline Parameter & \multicolumn{2}{c}{ No. (\%) } & p-value \\
\cline { 2 - 4 } & Low knowledge & Average knowledge & High knowledge \\
\hline Education level & & $3(16.7)$ & $1(5.6)$ \\
\hline No formal education/primary & $14(77.8)$ & $19(35.2)$ & $4(7.4)$ \\
\hline Secondary & $31(57.4)$ & $11(40.7)$ & $7(25.9)$ \\
\hline $\begin{array}{l}\text { A-level/diploma (ITE/polytechnic/ } \\
\text { private school)/university/post-tertiary }\end{array}$ & $9(33.3)$ & & \\
\hline
\end{tabular}

ITE: Institute of Technical Education; OPAAT: Osteoporosis Prevention and Awareness Tool

Table IV. Correlation between willingness to undergo BMD screening and knowledge of osteoporosis using the OPAAT.

\begin{tabular}{|llll|}
\hline Parameter & \multicolumn{2}{c}{ No. (\%) } & p-value \\
\cline { 2 - 3 } & $\begin{array}{l}\text { Willing to } \\
\text { be screened }\end{array}$ & $\begin{array}{l}\text { Not willing to } \\
\text { be screened }\end{array}$ & \\
\hline Knowledge score & & & 0.067 \\
\hline Low $(<19)$ & $30(55.6)$ & $24(44.4)$ & \\
\hline Average/high $(\geq 19)$ & $33(73.3)$ & $12(26.7)$ & \\
\hline
\end{tabular}

BMD: bone mineral density; OPAAT: Osteoporosis Prevention and Awareness Tool

However, participants showed a poorer understanding of the disease's pathophysiology and confused the symptoms with those of osteoarthritis.

Although it is suggestive that better osteoporosis knowledge corresponded to willingness to undergo osteoporosis screening, the results did not achieve statistical significance in this pilot study. The common barriers to BMD screening cited by the participants also suggested a lack of understanding of the disease and the screening process, affecting the screening rates. A larger-scale study or an educational intervention study ${ }^{(17)}$ would be useful to look for a statistically significant correlation between osteoporosis knowledge and screening rates in our population.

Incidentally, participants from Polyclinic A had a significantly higher rate of osteoporosis screening. However, there was no significant difference in their knowledge of osteoporosis. Beyond the scope of this study, a follow-up study can be considered to explore what motivated the participants from Polyclinic A to undergo osteoporosis screening.

A limitation of our study is that the questionnaire was only available in the English language, which may have created a bias in population selection for this study. Some participants may have had more difficulty comprehending the material, but this should have been addressed by having the study investigator being available to provide simple clarification if the participants had any questions. Follow-up studies are indicated to explore screening rates, knowledge of osteoporosis and barriers to screening in the non-English speaking and less-educated population, which may be more representative of the general population in Singapore.

Another limitation was that some of the participants took longer to complete the survey or were unable to complete the survey if they were called to attend the doctor's consultation, and the study investigator was unable to follow up with them after the consultation was complete.

Future studies may also investigate the impact of factors, such as OSTA score, FRAX score, existing functional status, physical activity level, and distance from home to public amenities and parks, on osteoporosis knowledge and willingness to undergo BMD screening.

In conclusion, osteoporosis and resultant fragility fractures have significant implications for the quality of life of our elderly patients and result in a significant economic burden on the healthcare system. The less-educated population also lacks osteoporosis knowledge, which leads to misconceptions about osteoporosis and the need for osteoporosis screening. Health education should rectify these common misconceptions of the disease, increase awareness of osteoporosis and improve screening rates.

\section{ACKNOWLEDGEMENTS}

We would like to appreciate Winnie Sea Sui Gek, Usha Sankari and Eileen Koh Yi Ling of the SingHealth Duke-NUS Family Medicine Academic Clinical Programme for their roles in study administration, data management and analysis for this study.

\section{REFERENCES}

1. Department of Statistics, Singapore. Population trends. Available at: https://www. singstat.gov.sg/publications/population/population-trends. Accessed November 15, 2020.

2. Yong EL, Ganesan G, Kramer MS, et al. Hip fractures in Singapore: ethnic differences and temporal trends in the new millennium. Osteoporos Int 2019; 30:879-86.

3. Demontiero O, Vidal C, Duque G. Aging and bone loss: new insights for the clinician. Ther Adv Musculoskelet Dis 2012; 4:61-76.

4. Ang SB. Approach and assessment of osteoporosis: identifying and managing risk. Singapore Fam Physician 2018; 44:7-10.

5. Yong EL, Ganesan G, Kramer MS, et al. Risk factors and trends associated with mortality among adults with hip fracture in Singapore. JAMA Netw Open 2020; 3:e1919706.

6. Chandran M, Lau TC, Gagnon-Arpin I, et al. The health and economic burden of osteoporotic fractures in Singapore and the potential impact of increasing treatment rates through more pharmacological options. Arch Osteoporos 2019; $14: 114$

7. Cheung EYN, Tan KCB, Cheung CL, Kung AWC. Osteoporosis in East Asia: current issues in assessment and management. Osteoporos Sarcopenia 2016; 2:118-33.

8. Saw SM, Hong CY, Lee J, et al. Awareness and health beliefs of women towards osteoporosis. Osteoporos Int 2003; 14:595-601.

9. Cosman F, de Beur SJ, LeBoff MS, et al. Erratum to: Clinician's guide to prevention and treatment of osteoporosis. Osteoporos Int 2015; 26:2045-7.

10. US Preventive Services Task Force; Curry SJ, Krist AH, Owens DK, et al. Screening for osteoporosis to prevent fractures: US Preventive Services Task Force recommendation statement. JAMA 2018; 319:2521-31.

11. Kung AWC, Wu CH, Itabashi A, et al. International Society for Clinical Densitometry official positions: Asia-Pacific Region consensus. J Clin Densitom 2010; 13:346-51.

12. Koh LK, Sedrine WB, Torralba TP, et al. A simple tool to identify Asian women at increased risk of osteoporosis. Osteoporos Int 2001; 12:699-705.

13. Chandran M, Chin YA, Choo KS, et al. Comparison of the Osteoporosis SelfAssessment Tool for Asians and the fracture risk assessment tool - FRAX to identify densitometric defined osteoporosis: a discriminatory value analysis in a multi-ethnic female population in Southeast Asia. Osteoporos Sarcopenia 2020; 6:53-8. 
14. Toh LS, Lai PSM, Wu DBC, et al. The development and validation of the Osteoporosis Prevention and Awareness Tool (OPAAT) in Malaysia. PLoS One 2015; 10:e0124553.

15. Department of Statistics, Singapore. Population and households. Available at: https://www.singstat.gov.sg/publications/reference/singapore-in-figures/ population-and-households. Accessed November 15, 2020.
16. Chandran M, Cheen M, Ying H, Lau TC, Tan M. Dropping the ball and falling off the care wagon. Factors correlating with nonadherence to secondary fracture prevention programs. J Clin Densitom 2016; 19:117-24.

17. Cranney A, Lam M, Ruhland L, et al. A multifaceted intervention to improve treatment of osteoporosis in postmenopausal women with wrist fractures: a cluster randomized trial. Osteoporos Int 2008; 19:1733-40 